Habib Ammari

\title{
An Introduction to
}

Mathematics of Emerging

Biomedical Imaging

Springer 


\section{Contents}

1 Biomedical Imaging Modalities …………………………………….... 3

1.1 X-Ray Imaging and Computed Tomography ……………………. 3

1.2 Magnetic Resonance Imaging …………………………………………... 4

1.3 Electrical Impedance Tomography ………………………………….. 5

1.4 T-Scan Electrical Impedance Imaging System for Anomaly

Detection

1.5 Electrical and Magnetic Source Imaging

1.6 Magnetic Resonance Electrical Impedance Tomography ........... 9

1.7 Impediography ...................................................................................... 10

1.8 Ultrasound Imaging ................................................................................. 11

1.9 Microwave Imaging …………………………………………………... 12

1.10 Elastic Imaging ....................................................................................... 12

1.11 Magnetic Resonance Elastography ........................................................ 12

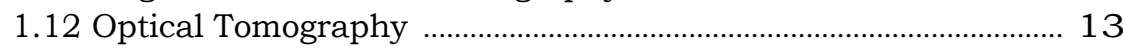

Part I Mathematical Tools

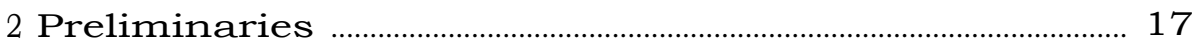

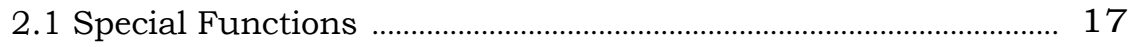

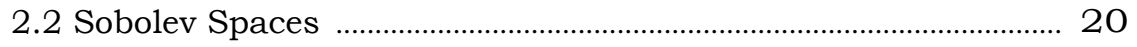

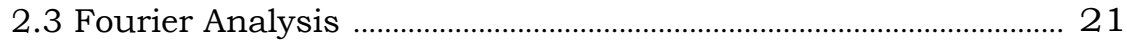

2.3.1 Shannon's Sampling Theorem ………………………………... 23

2.3.2 Fast Fourier Transform .............................................................. 24

2.4 The Two-Dimensional Radon Transform ………………………….. 25

2.5 The Moore-Penrose Generalized Inverse …………………………….. 28

2.6 Singular Value Decomposition ……………………………………… 28

2.7 Compact Operators ………………………………………………………. 29

2.8 Regularization of Ill-Posed Problems ………………………………... 30

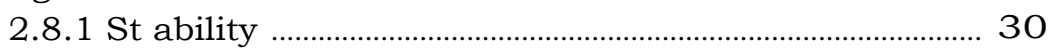

2.8.2 The Truncated SVD ................................................................ 32 
2.8.3 Tikhonov-Phillips Regularization ....................................... 32

2.8.4 Regularization by Truncated Iterative Methods ................ 34

2.9 General Image Characteristics ........................................................ 35

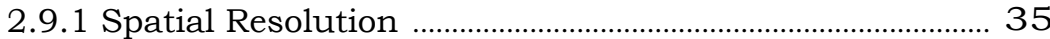

2.9.2 Signal-To-Noise Ratio ................................................................ 37

3 Layer Potential Techniques ............................................................ 43

3.1 The Laplace Equation .................................................................... 44

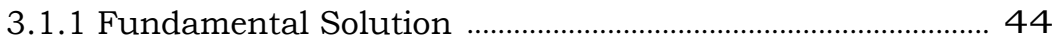

3.1.2 Layer Potentials ........................................................................... 46

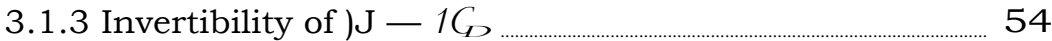

3.1.4 Neumann Function ................................................................. 55

3.1.5 Transmission Problem ………….............................................. 59

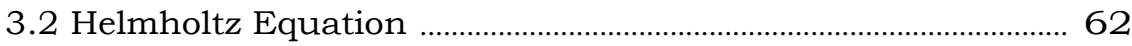

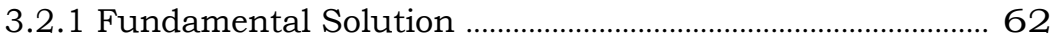

3.2.2 Layer Potentials ............................................................................... 63

3.2.3 Transmission Problem ……......................................................... 65

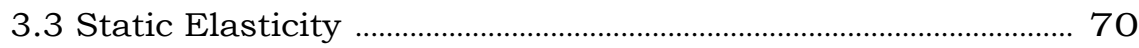

3.3.1 Fundamental Solution …........................................................ 71

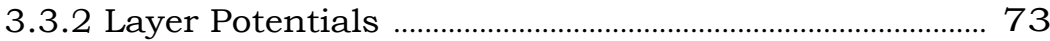

3.3.3 Transmission Problem ……………........................................ 75

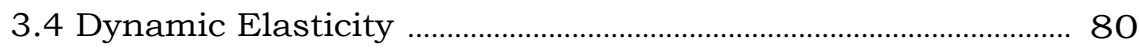

3.4.1 Radiation Condition …………........................................... 81

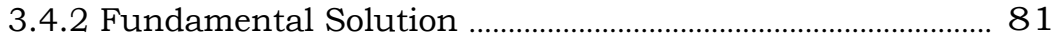

3.4.3 Layer Potentials ......................................................................... 82

3.4.4 Transmission Problem …………………………………….... 83

3.5 Modified Stokes System ......................................................................... 84

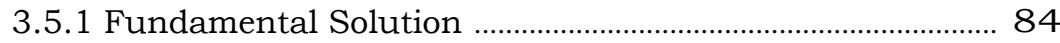

3.5.2 Layer Potentials .................................................................... 85

3.5.3 Transmission Problem ………………………………………. 89

\section{Part II General Reconstruction Algorithms}

4 Tomographic Imaging with Non-Diffracting Sources ........... 95

4.1 Imaging Equations of CT and MRI ................................................. 95

4.1.1 Imaging Equation of CT .................................................... 95

4.1.2 Imaging Equation of MRI ................................................ 96

4.2 General Issues of Image Reconstruction ........................................ 97

4.3 Reconstruction from Fourier Transform Samples ........................... 98

4.3.1 Problem Formulation ………….............................................. 98

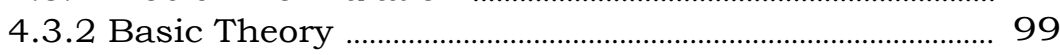

4.4 Reconstruction from Radon Transform Samples ............................ 101

4.4.1 The Inverse Radon Transform ……........................................... 101

4.4.2 Fourier Inversion Formula ................................................... 101 
4.4.3 Direct Backprojection Method 102

4.4.4 Filtered Backprojection Reconstruction ............................. 104

4.4.5 Noise in Filtered Backprojection Reconstruction .............. 105

5 Tomographic Imaging with Diffracting Sources ....................... 107

5.1 Electrical Impedance Tomography ………...................................... 107

5.1.1 Mathematical Model _............................................................ 108

5.1.2 Ill-Conditioning .................................................................. 108

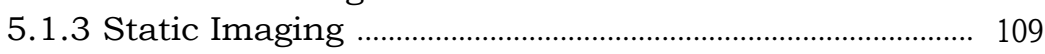

5.1.4 Dynamic Imaging .............................................................. 110

5.1.5 Electrode Model ......................................................................... 112

5.2 Ultrasound and Microwave Tomographies ......................................... 112

5.2.1 Mathematical Model .............................................................. 113

5.2.2 Diffraction Tomography …................................................... 114

6 Biomagnetic Source Imaging …...................................................... 117

6.1 Mathematical Models ...................................................................... 118

6.1.1 The Electric Forward Problem ………................................ 119

6.1.2 The Magnetic Forward Problem ………................................ 119

6.2 The Inverse EEG Problem .............................................................. 120

6.3 The Spherical Model in MEG ......................................................... 121

Part III Anomaly Detection Algorithms

7 Small Volume Expansions ..................................................................... 127

7.1 Conductivity Problem ...................................................................... 128

7.1.1 Formal Derivations .............................................................. 129

7.1.2 Polarization Tensor ……………….................................... 131

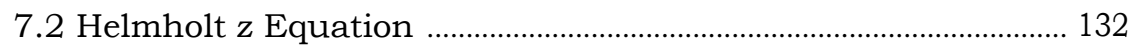

7.2.1 Formal Derivations ................................................................ 134

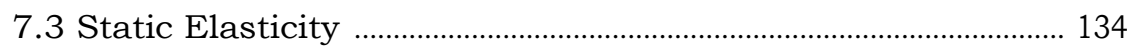

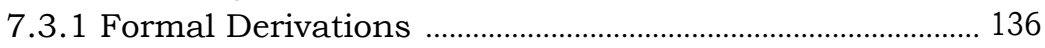

7.3.2 Elastic Moment Tensor ………….......................................... 138

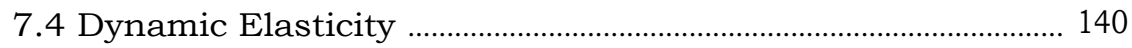

7.5 Modified Stokes System .................................................................. 140

7.6 Nearly Incompressible Bodies ................................................... 141

7.6.1 Formal Derivations ................................................................... 142

7.6.2 Viscous Moment Tensor ………………................................. 145

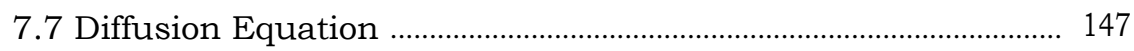

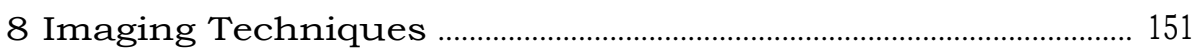

8.1 Projection Type Algorithms ……................................................. 151

8.2 Multiple Signal Classification Type Algorithms ........................... 152

8.3 Time-Domain Imaging ............................................................. 156 
Part IV Hybrid Imaging Techniques

9 Magnetic Resonance Electrical Impedance Tomography ....... 169

9.1 Mathematical Model …...................................................................... 170

9.2 J-Substitution Algorithm ............................................................... 172

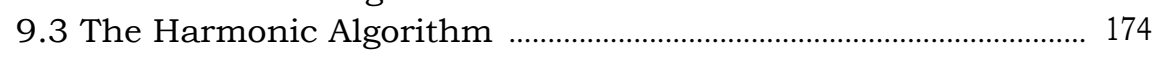

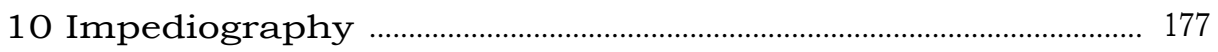

10.1 Physical Model ................................................................................ 177

10.2 Mathematical Model …...................................................................... 178

10.3 E-Substitution Algorithm ........................................................... 180

11 Magnetic Resonance Elastography .................................................. 183

11.1 Mathematical Model ......................................................................... 183

11.2 Binary Level Set Algorithm .......................................................... 185

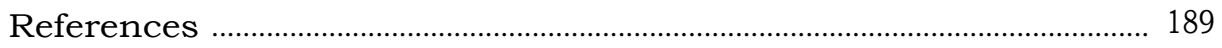

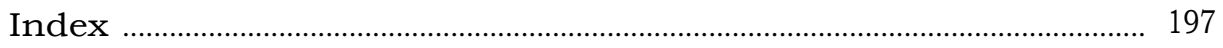

